\title{
Annual Journal Citation Indices: A Comparative Study
}

\author{
Abdul Khaleque ${ }^{1, *}$, Arnab Chatterjee ${ }^{2, \dagger}$, Parongama Sen ${ }^{1, \neq}$ \\ ${ }^{1}$ Department of Physics, University of Calcutta, 92 A P C Road, Kolkata 700 009, India \\ ${ }^{2}$ Condensed Matter Physics Division, Saha Institute of Nuclear Physics, A/AF Bidhannagar, Kolkata 700 064, India
}

\begin{abstract}
We study the statistics of citations made to the indexed Science journals in the Journal Citation Reports during the period 2004-2013 using different measures. We consider different measures which quantify the impact of the journals. To our surprise, we find that the apparently uncorrelated measures, even when defined in an arbitrary manner, show strong correlations. This is checked over all the years considered. Impact factor being one of these measures, the present work raises the question whether it is actually a nearly perfect index as claimed often. In addition, we study the distributions of the different indices which also behave similarly.
\end{abstract}

Keywords: Bibliometrics, Citation, Impact factor, Citation rate, Correlations, Ageing.

\section{INTRODUCTION}

Extensive studies have been made to analyze quantitatively the popularity of commodities (e.g., books, DVDs), movies, academic publications, webpages etc. over the last two decades or so, thanks to the availability of such data. Remarkably, identical behaviour of the relevant distributions have been observed in many cases suggesting a common dynamical scheme responsible for the universality. To study the popularity of a research publication or paper, usually one calculates the citations made to that paper. The citation probability over time as well as citation distributions have been studied in great detail in recent years. ${ }^{[1-13]}$ The popularity of an academic journal may also be similarly quantified using the citation data made to the papers published therein. The total citations received in a year, the impact factor, ${ }^{[14,15]}$ and the eigenfactor ${ }^{[16]}$ are

*Address for correspondence:

*E-mail: aktphys@gmail.com.

†Email: arnabchat@gmail.com

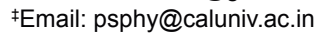

\begin{tabular}{|c|c|}
\hline \multicolumn{2}{|c|}{ Access this article online } \\
\hline Official Publication of & \\
\hline & $\begin{array}{l}\text { Website: } \\
\text { www.jscires.org }\end{array}$ \\
\hline 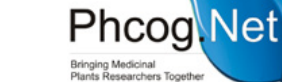 & $\begin{array}{l}\text { DOI: } \\
\text { 10.5530/jscires.5.1.5 }\end{array}$ \\
\hline
\end{tabular}

well-known popular measures. The impact factor (IF) ${ }^{[14,15]}$ of an academic journal is a measure which reflects the average number of citations to recent articles published in the same journal. It is frequently used as a proxy for the relative importance of a journal within its field, with journals with higher IFs deemed to be more important compared to those with lower ones. However, according to, ${ }^{[17]}$ IF may not be the perfect measure to compare the quality of two journals. The eigen factor measure in addition takes into account the quality of the journals in which the citing articles appear, arguing that a journal is considered to be more influential if it is cited often by other influential journals. It was shown ${ }^{[18]}$ however that the eigenfactor measurement is more or less correlated with the annual citation measure.

Apart from studying the properties/statistics of the standard measures of annual citation and impact factor, we also introduce and analyze a new measure called the citation rate, defined in the next section. 
In the present paper, we analyze the inter-dependence of the three indices, correlations of the same measures over time (auto-correlations), as well as their distributions. In section 2, we define the quantities considered: the details of the data and results are presented in section 3 and in the last section summary and discussions are made.

\section{DEFINITION OF CITATION INDICES}

Impact factors are calculated yearly for journals that are indexed in the Journal Citation Reports. ${ }^{[19]}$ The precise definition of IF is the following: if papers published in a journal in years $T-2$ and $T-1$ are cited $N(T-2)+N(T-1)$ times by indexed journals in the year $T$, and $N(T-2)+N$ $(T-1)$ be the number of citable articles published in those years, then the impact factor in year $T$ is given by

$$
I(T) \frac{N(T-2)+N(T-1)}{N(T-2)+N(T-1)}
$$

One can also measure $n(T)$, the number of annual citations (AC) to a journal in a given year. This is given by

$$
n(T)=\sum_{t \leq T} \sum_{i} A_{i}(t, T)
$$

where $A_{i}(t, T)$ is the citations received in the year $T$ by the $i$ th paper published in the year $t \leq T$.

We calculate another index, $r(T)$, the annual citation rate (CR) at a particular year $T$ that is defined as the number of citations received in a year (annual citations) divided by the number of articles published in the same year. Formally,

$$
r(T)=\frac{n(T)}{N(T)}
$$

Note that this is clearly different from the average citation rate defined in $^{[20]}$ which denotes average number of citations received in a particular time interval by all previously published papers. However it is rather arbitrary as the numerator and the denominator are uncorrelated. We introduce this measure with the purpose to see how important is this arbitrariness.

These three measures are available from a single year's report citation data. Combining data of different years, we consider another index $r$ ' which may be less arbitrary than $r$ but still quite different from $I$. We define $r$ as $r^{\prime}(T)=n(T) /\langle N(T)>$, where $\mathrm{N}$ is the average of $\langle N(T)\rangle$ over a extended time interval (10 years in our case).
The number of annual citations $n(T)$ might depend on the age of the journal as well as on the number of papers published in it. Detailed studies on citation data have shown that a paper's citation probability decays with time as a power law roughly up to 20 years after its publication after which it falls drastically. ${ }^{[21]}$ So, one can assume that the total citation $n(T)$ consists of citation to papers not more than $\sim 20$ years old practically. Hence, if the ages of the journals considered are greater than $\sim 20$ mostly, $n(T)$ approximately covers the same time period for all journals and age of a journal will not be an important factor. However, $n(T)$ may be biased by the number of publications and thus it is meaningful to scale it by a typical number of publications (as done for $r$ and $r$ ).

\section{DATA AND RESULTS}

We collected data for all Science journals indexed in ISI Web of Knowledge for the Science database, containing the following information: (i) the number of citations $n(T)$ received by the journal in a year $T$ (ii) IF $I(T)$ in that year $T$, (iii) number of papers $N(T)$ published in that particular year $T$. The data is for 10 years (2004-2013) taken from Journal Citation Reports (JCR). ${ }^{[1]}$

\section{Correlations}

We first report the correlation between the different measures for different years. Figure 1 and 2 show the behavior of $I$ versus $n$ and $r$ respectively. The impact factor $I$ shows remarkable correlation with the number of citations $n$ for each year. In fact, the data binned for number of citations shows a very good agreement with a power law: $I=a n^{\xi_{n}}$ with $\xi_{n}=0.47 \pm 0.03$ considering all the years. The values of the exponents $a$ and $\xi_{n}$ for different years are given in Table 1. The binned data in Figure 2 indicate that $I$ and $r$ are also related by a power law but there are apparently two distinct scaling regimes, roughly below and above $r \approx 50$. Fitting the data piecewise by power laws, we get $I \propto r^{\xi_{r}}$ with $\xi_{r 1}=0.60 \pm 0.02$ for $\mathrm{r}<50$ and $\xi_{r 2}=1.09 \pm 0.03$ otherwise for 2004 and $\xi_{r 1}=0.55 \pm 0.02$ for $r<50$ and $\xi_{r 2}=0.89 \pm 0.08$ otherwise for 2013. The power law exponent for the low $\mathrm{r}$ region is less than that in the high $r$-a trend that is consistent for all years, except that the exponents are slightly different (see Table 2 for all years). Figure 3 shows the variation of $r$ with $n$, from where it is quite interesting to note that the annual citations and citation rates have a different functional dependence. Here, the variation of $r$ with $n$ fitted well with the form $r=\exp \left(c_{n}+a_{n}(\log n)^{b_{n}}\right)$ and the estimated exponents are tabulated in Table 
Table 1: Table for different Table for the value of the exponents $a$ and $\xi_{n}$ years. The fitted form is $I=a n_{n}$

\begin{tabular}{ccc}
\hline Year & $a$ & $\boldsymbol{\xi}_{n}$ \\
\hline 2004 & $0.04 \pm 0.01$ & $0.50 \pm 0.02$ \\
2005 & $0.04 \pm 0.01$ & $0.47 \pm 0.01$ \\
2006 & $0.05 \pm 0.01$ & $0.49 \pm 0.01$ \\
2007 & $0.05 \pm 0.01$ & $0.47 \pm 0.01$ \\
2008 & $0.06 \pm 0.02$ & $0.46 \pm 0.02$ \\
2009 & $0.06 \pm 0.03$ & $0.43 \pm 0.03$ \\
2010 & $0.06 \pm 0.01$ & $0.45 \pm 0.02$ \\
2011 & $0.07 \pm 0.05$ & $0.44 \pm 0.03$ \\
2012 & $0.05 \pm 0.03$ & $0.46 \pm 0.02$ \\
2013 & $0.04 \pm 0.03$ & $0.49 \pm 0.02$ \\
\hline
\end{tabular}

Table 2: Table for the value of the Exponents a and $\xi_{r}$ for different years. The fitted form is $I=a n^{\xi}$.

\begin{tabular}{ccccc}
\hline Year & $\boldsymbol{a}$ for low $\boldsymbol{r}$ & $\boldsymbol{\xi}_{\boldsymbol{r}}$ for low $\boldsymbol{r}$ & $\boldsymbol{a}$ for high $\boldsymbol{r}$ & $\boldsymbol{\xi}_{\boldsymbol{r} 2}$ for high $\boldsymbol{r}$ \\
\hline 2004 & $0.26 \pm 0.01$ & $0.60 \pm 0.02$ & $0.03 \pm 0.02$ & $1.10 \pm 0.03$ \\
2005 & $0.24 \pm 0.01$ & $0.66 \pm 0.01$ & $0.09 \pm 0.04$ & $0.93 \pm 0.09$ \\
2006 & $0.28 \pm 0.01$ & $0.61 \pm 0.01$ & $0.07 \pm 0.05$ & $0.95 \pm 0.13$ \\
2007 & $0.28 \pm 0.02$ & $0.62 \pm 0.01$ & $0.06 \pm 0.06$ & $0.98 \pm 0.16$ \\
2008 & $0.26 \pm 0.03$ & $0.65 \pm 0.03$ & $0.04 \pm 0.02$ & $1.07 \pm 0.09$ \\
2009 & $0.29 \pm 0.02$ & $0.62 \pm 0.02$ & $0.11 \pm 0.05$ & $0.86 \pm 0.08$ \\
2010 & $0.29 \pm 0.02$ & $0.64 \pm 0.03$ & $0.06 \pm 0.03$ & $1.00 \pm 0.09$ \\
2011 & $0.31 \pm 0.03$ & $0.61 \pm 0.02$ & $0.11 \pm 0.03$ & $0.87 \pm 0.05$ \\
2012 & $0.31 \pm 0.02$ & $0.60 \pm 0.02$ & $0.04 \pm 0.01$ & $1.05 \pm 0.06$ \\
2013 & $0.36 \pm 0.02$ & $0.55 \pm 0.02$ & $0.10 \pm 0.04$ & $0.89 \pm 0.08$ \\
\hline
\end{tabular}

Table 3: Table for $a, b$, and $c$ for different years. The fitted form is $r=\exp \left(c+a(\log n)^{b}\right)$

\begin{tabular}{cccc} 
Year & $\boldsymbol{a}$ & $\boldsymbol{b}$ & $\boldsymbol{c}$ \\
\hline 2004 & $4.32 \pm 1.35$ & $0.40 \pm 0.08$ & $-6.59 \pm 1.44$ \\
2005 & $4.84 \pm 1.66$ & $0.38 \pm 0.08$ & $-7.23 \pm 1.78$ \\
2006 & $7.26 \pm 1.96$ & $0.29 \pm 0.06$ & $-9.86 \pm 2.01$ \\
2007 & $3.22 \pm 1.14$ & $0.48 \pm 0.10$ & $-5.42 \pm 1.28$ \\
2008 & $3.27 \pm 0.85$ & $0.48 \pm 0.08$ & $-5.53 \pm 0.91$ \\
2009 & $2.90 \pm 0.58$ & $0.50 \pm 0.06$ & $-4.87 \pm 0.64$ \\
2010 & $1.88 \pm 0.48$ & $0.63 \pm 0.08$ & $-3.58 \pm 0.58$ \\
2011 & $3.67 \pm 0.70$ & $0.45 \pm 0.05$ & $-5.96 \pm 0.77$ \\
2012 & $1.38 \pm 0.33$ & $0.73 \pm 0.08$ & $-2.88 \pm 0.42$ \\
2013 & $6.29 \pm 1.19$ & $0.33 \pm 0.04$ & $-8.94 \pm 1.23$ \\
\hline
\end{tabular}

3. The most relevant exponent $b_{n}$ has a value roughly around 0.5 with some variation for different years. It is interesting to find that annual citation rate $r$ which is an implicit function of the annual citations $\mathrm{n}$ has a nontrivial dependence.

\section{Auto-correlation}

We have also calculated the dynamic correlation of each of the indices $(n, I, r)$ with itself over consecutive years. Plotting the values for two different years for the same journal, the auto-correlation is estimated by calculating the correlation coefficient. The linear correlation coef-
Table 4: Table for the value of the correlation coefficient $\boldsymbol{R}$ for large $\boldsymbol{r}$ for all the pairs of consecutive years

\begin{tabular}{cc}
\hline Pairs of year & $\boldsymbol{R}$ \\
\hline $2004-2005$ & 0.7923 \\
$2005-2006$ & 0.8680 \\
$2006-2007$ & 0.8177 \\
$2007-2008$ & 0.7543 \\
$2008-2009$ & 0.9426 \\
$2009-2010$ & 0.9039 \\
$2010-2011$ & 0.9040 \\
$2011-2012$ & 0.9297 \\
$2012-2013$ & 0.9327 \\
\hline
\end{tabular}

ficient is a measure of the strength of linear relation between two quantitative variables, say $x_{i}$ and $y_{i}$. We use $\mathrm{R}$ to denote the sample correlation coefficient:

$$
R=\frac{\sum_{i=1}^{K}\left(x_{i}-\bar{x}\right)\left(y_{i}-\bar{y}\right)}{\sqrt{\sum_{i=1}^{K}\left(x_{i}-\bar{x}\right)^{2} \sum_{i=1}^{K}\left(y_{i}-\bar{y}\right)^{2}}}
$$

Where $K$ is the number of individuals in the sample.

In Figure 4, the correlations for $n$ and $I$ are presented. We choose two consecutive years from the extreme ends, i.e., 2004-05 and 2012-13. It is observed that these are highly correlated as $R$ is close to 1 in all the cases. Such high correlations are apparently not present for $r$ for all pairs of consecutive years. In fact, $R$ for $r$ shows considerable fluctuation as shown in Table 4. There may be an upward trend although from the last few data points there seems to be a tendency to stabilize at values which are still not very close to unity (compared to the correlation coefficient for $n$ and $I$ ). The lack of strong correlation in $r$ signifies the fluctuation in the number of publications even for consecutive years.

Apart from estimating the data for consecutive years, we have also calculated the correlation for the two extreme years for which data is available. The correlations for $n, I$ and $r$ of two extreme years 2004 and 2013 are shown in Figure 5. The value of $R$ in this case is still close to unity for $n$ while for $I$ and $r$ it is much less. That $\mathrm{R}$ is close to 1 for $n$ over a comparatively long time interval ( 9 years) is not surprising as for $\mathrm{n}$ citations to all previously published papers are counted. On the other hand, for $I$, the correlation drops since citations made for papers published two years prior to 2004 and that to 2013 are completely uncorrelated. The data for $r$ is not at all surprising as we already observed that even for consecutive years, correlation is not large. 
The correlation between $r$ and $r$ 'is found to be quite high. In Figure 6, we show the correlation between $\mathrm{r}$ and $\mathrm{r}$ for two years, $\mathrm{R}=0.9308$ for 2004 and $\mathrm{R}=0.9549$ for 2013 .

Distribution of annual citations, IF and annual citation rate: nature of their tails

First we investigate the nature of the tail of the distribution of annual citations $P(n)$ (Figure 7A and B) and impact factors Q(I) (Figure 7C and D). The plots showed excellent scaling collapse over years when in general for any probability distribution $X(x), X(x)<x>$ is plotted against $x /\langle x\rangle$. The distribution of annual citations and impact factors show non-monotonic behavior, with a peak occurring approximately at half the average values. The tail of the annual citations distributions (Figure 7(B)) fit well to a lognormal form

$$
\left(X(x)=\frac{1}{x \sigma \sqrt{2} \pi} \mathrm{e} \frac{(\log x-\mu)^{2}}{2 \sigma^{2}}\right)
$$

with $\mu=-1.355$ and $\mathrm{s}=1.573$. However, the tail of the impact factor distribution fits to a power law, with a decay exponent about $\gamma_{I}=2.92$.

The probability distributions $\Omega(r)$ of the newly proposed quantity, the annual citation rate $r$ also shows a power law tail with a decay exponent $\gamma_{\mathrm{r}} \approx 2.54$ (Figure 8B); although it is almost a flat distribution for $r /<r><1$. The probability distribution $\Omega\left(r^{\prime}\right)$ shows similar features as $\Omega(r)$ and its tail resembles roughly a power law with decay exponent $\gamma_{r^{\prime}} \approx 2.63$ (Figure 8D). In fact, we checked the correlation between $r$ and $r^{\prime}$ for each year, and found them to be very strongly correlated $(R>0.9)$.

If I and $r$ are related by a power law, one can in principle derive the exponent of the distribution of $r$ from that of $I$. Assuming in general the scaled distribution of $I$ has a power law tail with exponent $\gamma_{I}$ and $I \propto r^{r^{*}}$, the tail of the distribution for the scaled $r$ should follow the behavior $\left(r /<r>r^{2}+\xi^{2}-1\right)$. However, we have noted earlier that $\xi_{r}$ is not unique. Putting the value of $\gamma_{I}=2.92$ and the observed value $\gamma_{r}=2.54$, we obtain $\xi_{r} \approx 0.80$. It is interesting to note that this value does not correspond to either of the two values of $\xi_{r}$ (see Table. 2) estimated from the I- $r$ curves but rather is very close to the average of the two values in general.

\section{SUMMARY AND DISCUSSIONS}

We analyzed the citation data to Science journals for the period 2004-13 considering the entire data set available in the Journal Citation Reports. ${ }^{[19]}$ The analysis is based on four different measures or indices. While the impact factor $I$ and annual citation $n$ are readily available, we also introduced a third index, the citation rate $r$, which can be easily estimated from the database. Closely related to $r$ is another measure $r^{\prime}$ which is calculated combining different years.

We have primarily studied the correlation between different measures in the same year, correlation between the same measures in two different years and probability distribution functions for different years. In this paper, we reported the explicit functional forms by which any pair of the three $-I, n$ and $r$ are related to each other. Also correlation between $r$ and $r^{\prime}$ has been studied. The most surprising result is perhaps the fairly strong dependence of $I$ on $n$. This dependence is not accidental as the corresponding exponent does not show appreciable change over time. It is to be remembered that $I$ depends on the citations to recent publications only while $n$ considers citations to all published papers in a journal. One might expect that old journals will have larger value of $n$ as a result. Impact factor $I$ on the other hand will not depend on the age of the journal, in principle. Thus it is quite surprising to see that $I$ and $n$ show a strong functional dependence. The measure $r$ is also expected to be dependent on the age. However, in contrast to $n$ it is a scaled data as $I$, albeit in a different way, and it is not surprising that $I$ depends on $r$ in a stronger manner. However $r$ being quite arbitrarily defined, the correlation between $I$ and $r$ is not apparent. In fact, the numerator and denominator of $r$ are completely independent variables unless the number of publications is same for all years which is not the case. For $I$ on the other hand, the quantities appearing in the numerator and denominator are directly correlated. This work therefore opens up the question whether the impact factor is the most reliable measure or not. If so, then the present work indicates that $n$ and $r$ might as well qualify as such measures, which is less than obvious.

The probability distributions of the three measures are found to show conventional behavior, i.e., either log-normal type or they occur with power law tails. Like many other real systems, the exponents for power law variation lie between 2 and 3 which is sensible so as to have a finite average value. The scaled distribution of different years show universal behavior in time, as has been observed in many other systems in which the dynamics of popularity has been studied. ${ }^{[2]}$ The preference of lognormals and power laws are decided from eye estimates of best fits. However, the reason why annual citations may follow a lognormal distribution can be justified by the fact that annual citations are very highly correlated, and one can imagine an underlying multiplicative process. 

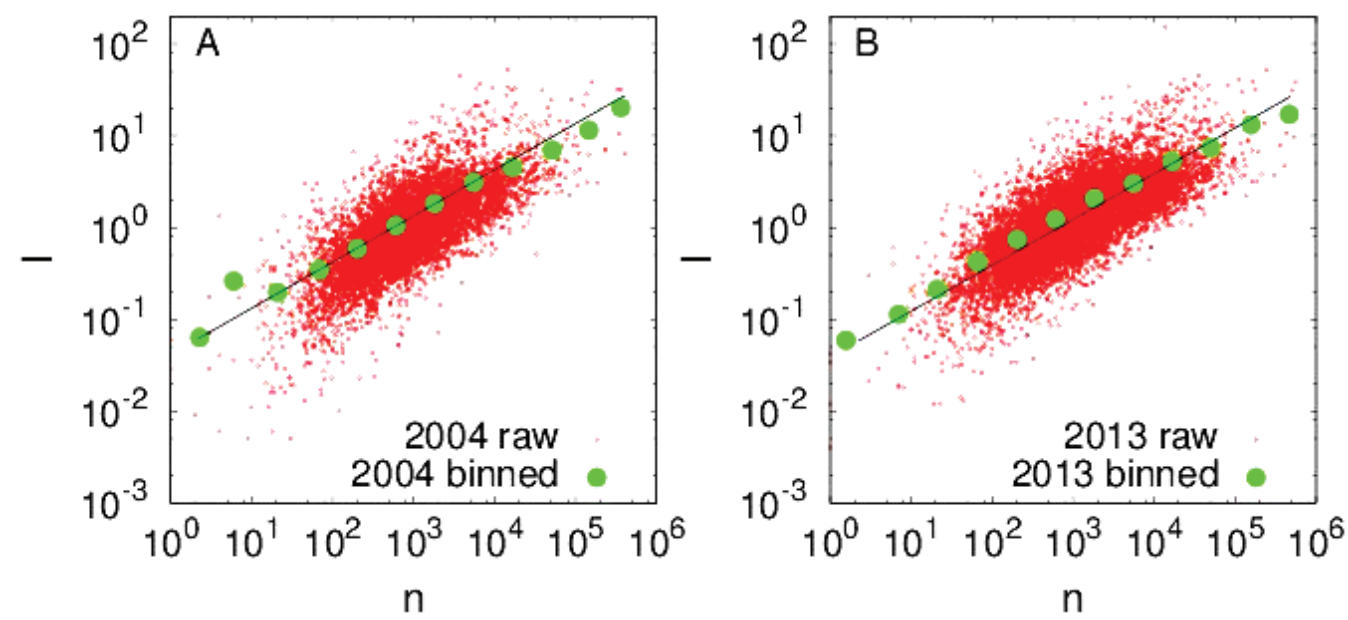

Figure 1: Scatter plot of impact factor (I) versus citation (n). The binned data are also shown, which seems to fit reasonably to $I \propto n^{\xi n}$. (a) The exponent $\xi_{\mathrm{n}} \approx 0.50$ for 2004 and (b) $\xi_{\mathrm{n}} \approx 0.49$ for 2013 .
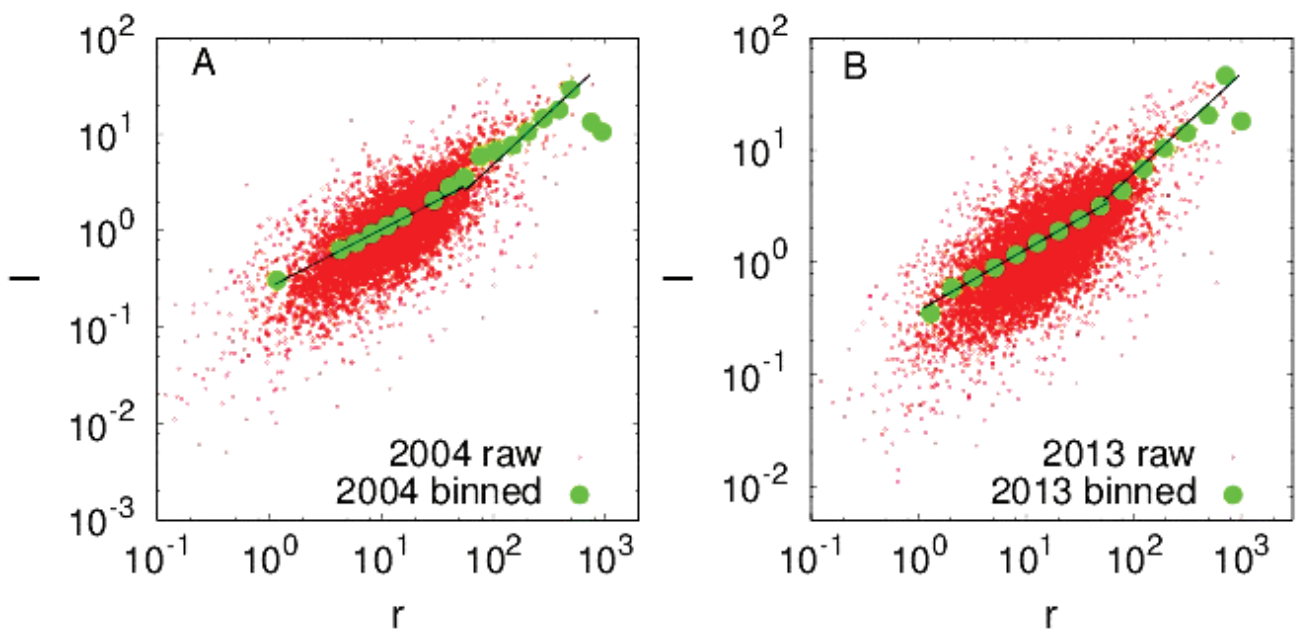

Figure 2: Scatter plot of impact factor (I) versus citation rate (r). The binned data are also shown, which seems to fit to $I \propto r^{\zeta r}$ with two different exponents. (a) For $2004, \xi_{\mathrm{r} 1} \approx 0.60$ for lower $\mathrm{r}$ value and $\xi_{\mathrm{r} 2} \approx 1.08$ for larger $\mathrm{r}$ value. (b) For $2013, \xi_{\mathrm{r} 1} \approx 0.55$ for lower $\mathrm{r}$ value and $\xi_{\mathrm{r} 2} \approx 0.89$ for larger $\mathrm{r}$ value.
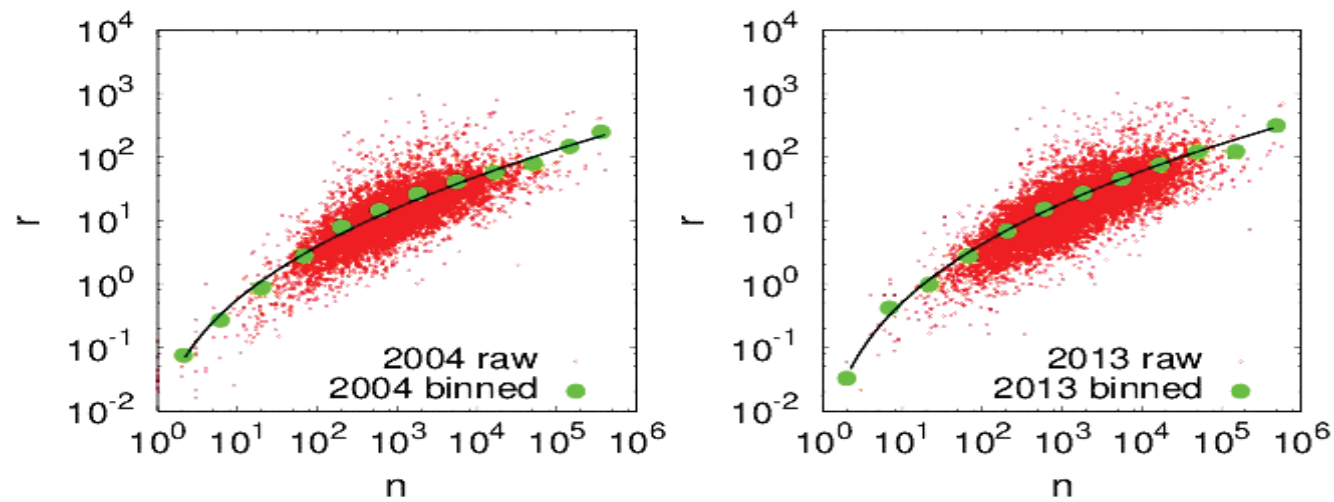

Figure 3: Scatter plot of citation rate (r) versus citation (n). The binned data are also shown, which fits well to $r=\exp \left(c_{n}+a_{n}(l o g\right.$ $\mathrm{n})^{\mathrm{bn}}$ ). (a) For 2004, the exponents are $\mathrm{a}_{\mathrm{n}} \sim 4.32, \mathrm{~b}_{\mathrm{n}} \sim 0.39$ and $\mathrm{c}_{\mathrm{n}} \sim-6.59$. (b) For 2013, the exponents are $\mathrm{a}_{\mathrm{n}} \sim 6.29, \mathrm{~b}_{\mathrm{n}} \sim 0.33$, and $\mathrm{c}_{\mathrm{n}}$ $\sim-8.93$. Details of fitting parameters for different years are shown in Table 3. 

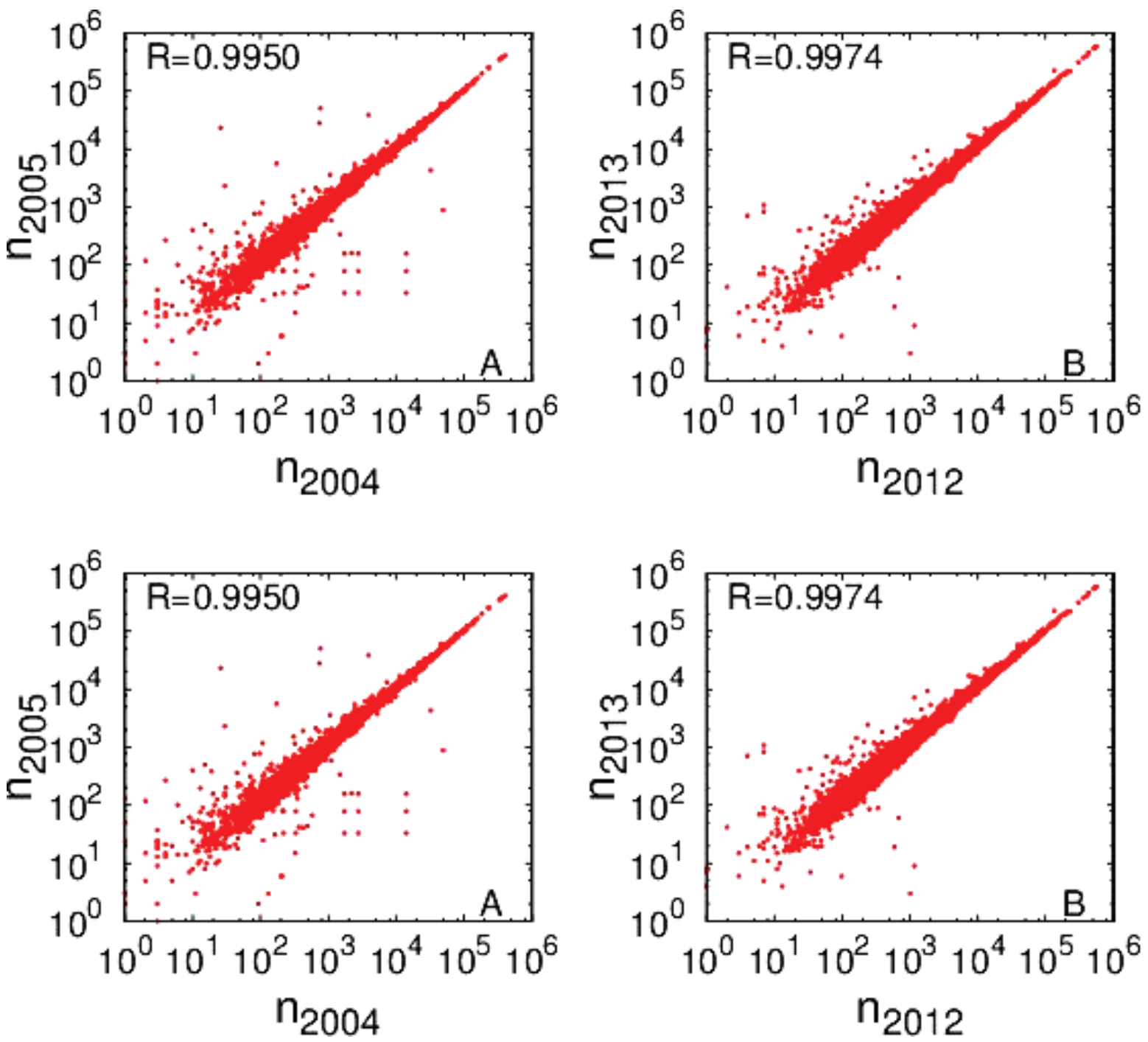

Figure 4: Correlation of actual values of annual citations n for two pairs of successive years (a) 2004-2005 and (b) 2012-2013. The correlation coefficient shows very high values, 0.9950 and 0.9974 , respectively. Correlation of actual values of impact factors I for two pairs of successive years (c) 2004-2005 and (d) 2012-2013. The correlation coefficient shows fairly high values, 0.9725 and 0.9838 , respectively.
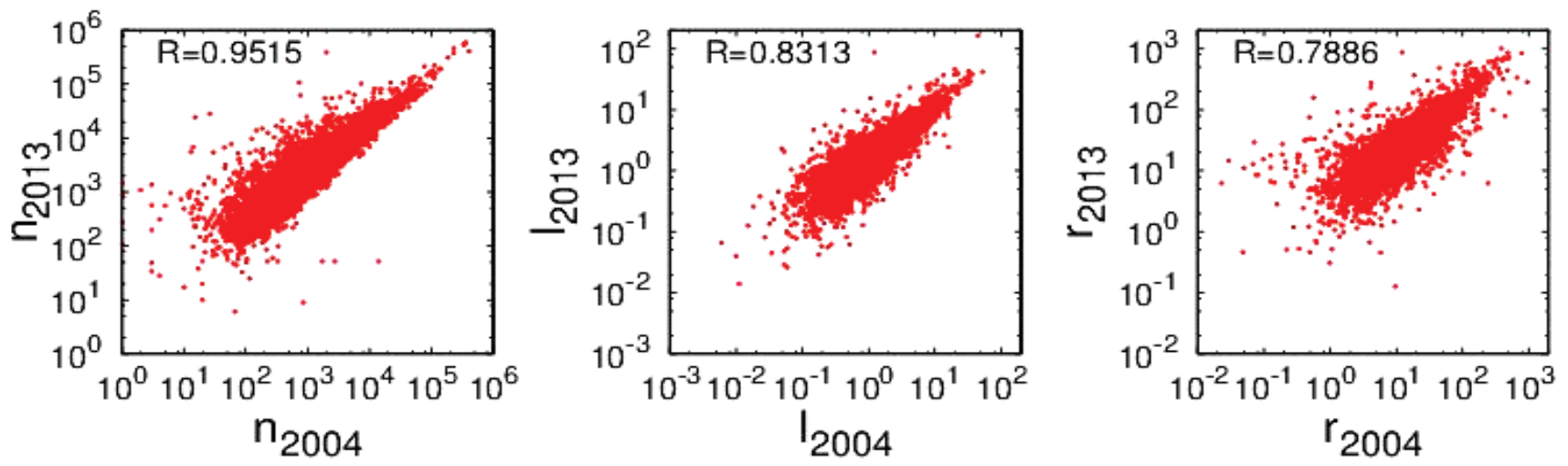

Figure 5: Correlation of actual values of (a) annual citations $\mathrm{n}$ for the pair of years 2004-2013. The correlation coefficient is 0.9515. (b) Same for impact factors I for the pair of years 2004-2013. The correlation coefficient is 0.8313. (c) Same for citation rates $\mathrm{r}$ for the pair of years 2004-2013. The correlation coefficient is 0.7886 . 

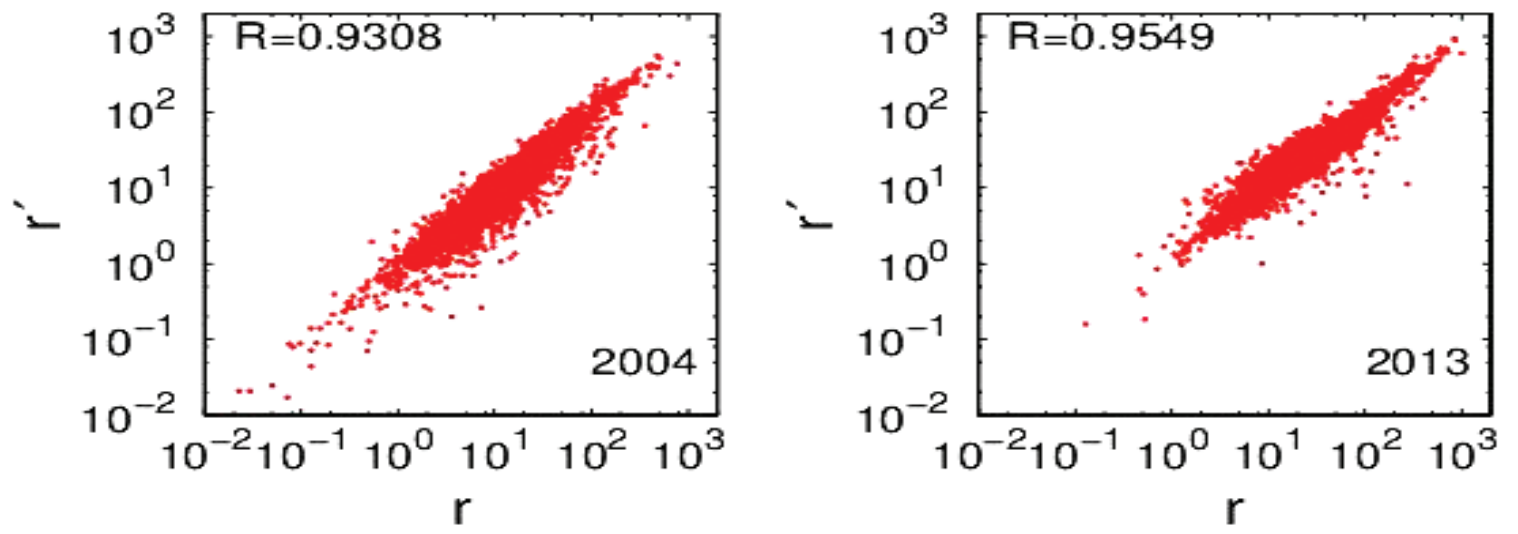

Figure 6: Correlation of $r$ and $r^{2}$ for $2004(\mathrm{R}=0.9308)$ and $2013(\mathrm{R}=0.9549)$.
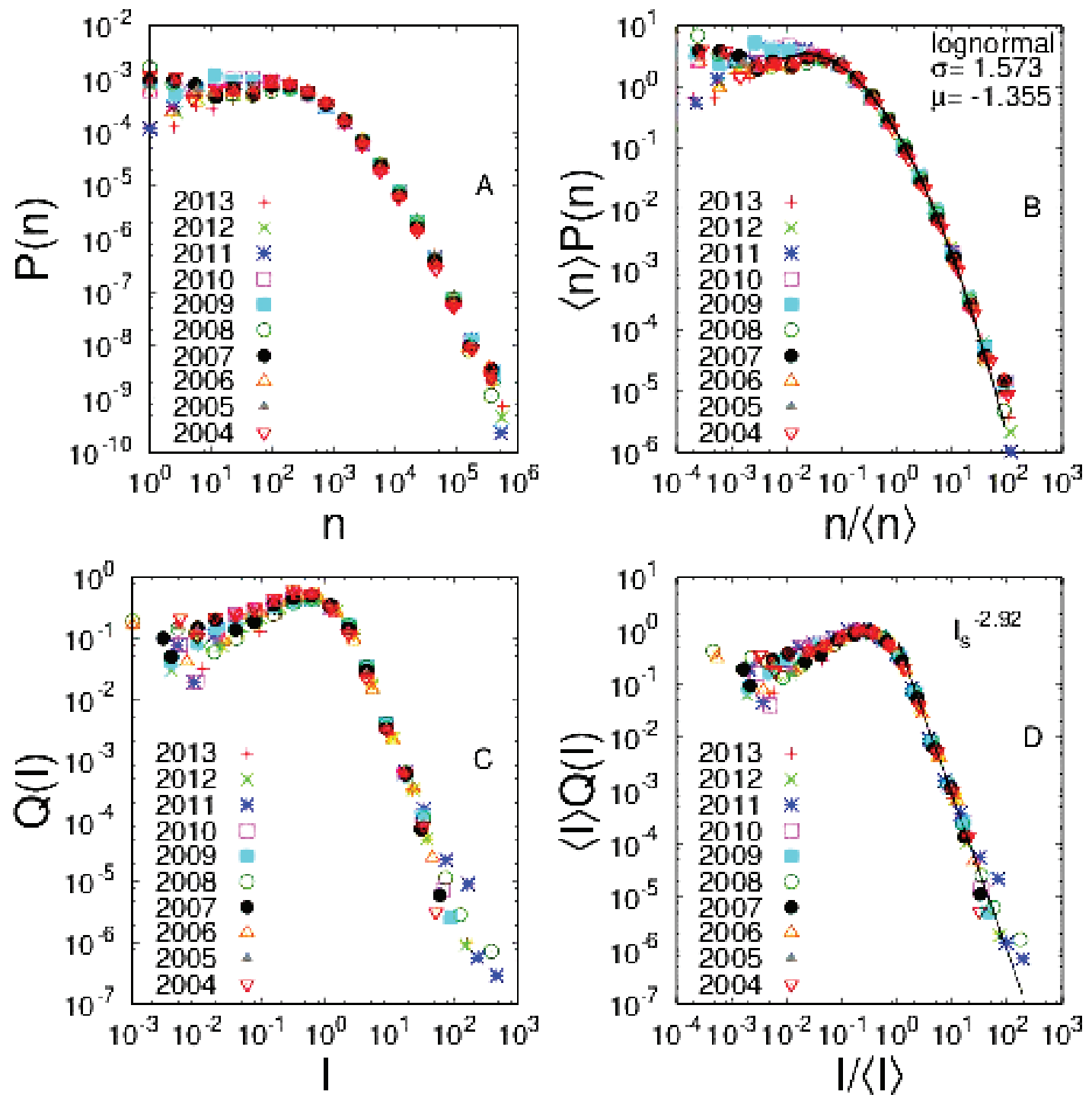

Figure 7: (a) The probability distribution of annual citations $P(n)$ and (b) scaling collapse of the same, which fits fairly well to a lognormal form. (c) The probability distribution of impact factor $\mathcal{Q}(I)$ and (d) scaling collapse of the same, the tail fits fairly well to a power law form. The straight line has slope $\gamma=2.92$. 

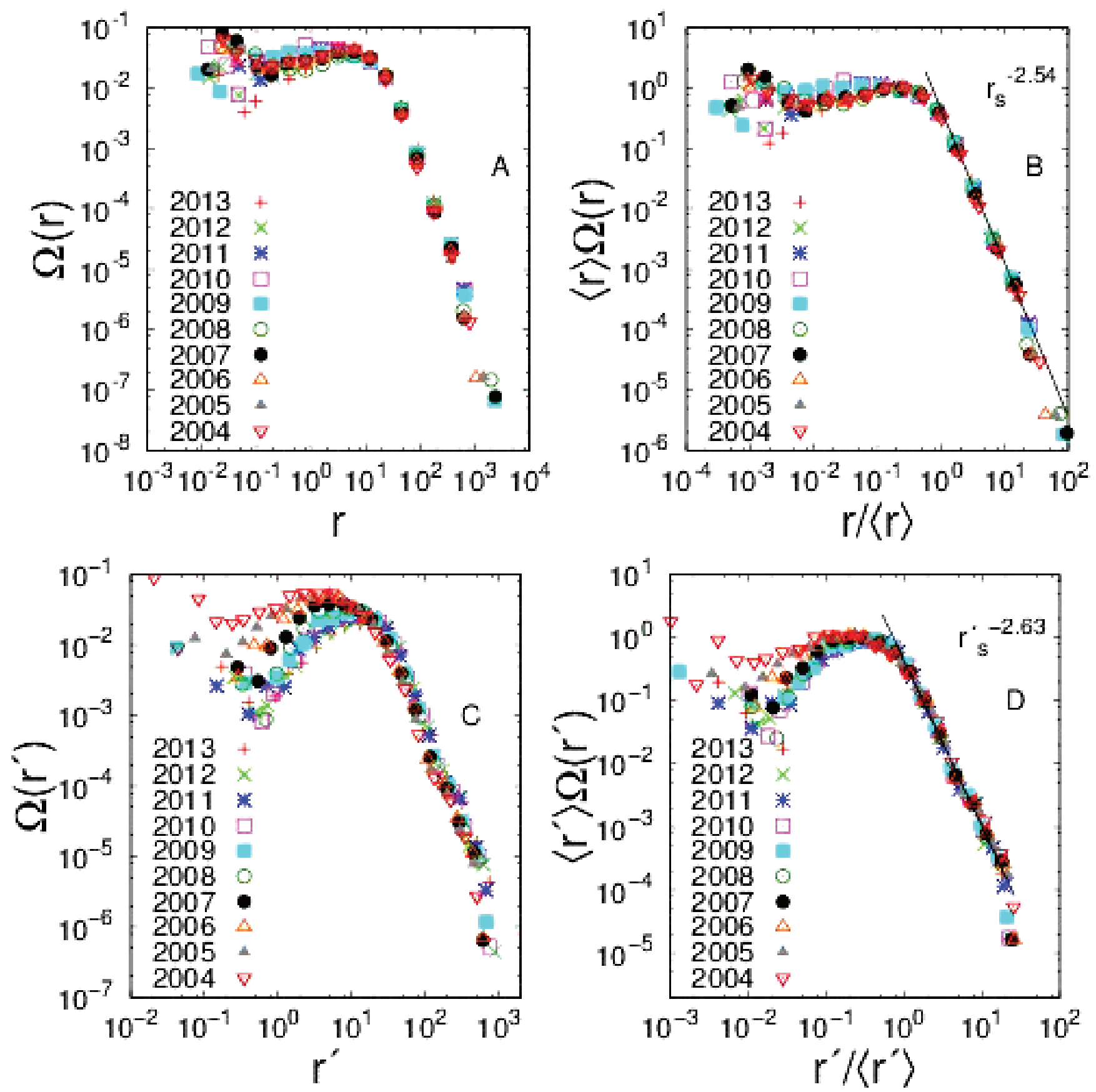

Figure 8: (a) The probability distribution of annual citation rates $(\Omega)(r)$ and (b) scaling collapse of the same, the tail fits fairly well to a power law form with a decay exponent $\gamma=2.54$. (c) The probability distribution of annual citation rates $(\Omega)\left(r^{r}\right)$ and $(\mathrm{d})$ scaling collapse of the same, the tail fits to a power law form with a decay exponent $\gamma_{r}=2.63 r^{2}$ being calculated using the average number of publications for the last 10 years.

\section{ACKNOWLEDGEMENT}

The Authors thank A. Ghosh for assisting in data acquisition. AK acknowledges financial support from UGC sanction No. F.7-48/2007(BSR). PS acknowledges financial support from CSIR project.

\section{REFERENCES}

1. Sen P, Chakrabarti BK. Sociophysics: An Introduction. Oxford: Oxford University Press; 2013.

2. Shockley W. On the statistics of individual variations of productivity in research laboratories. Proc IRE. 1957;45(3):27990. 
3. Laherrere J, Sornette D. Stretched exponential distributions in nature and economy:"fat tails" with characteristic scales. Eur Phys J B. 1998;2(4):525-39.

4. Redner S. How popular is your paper? An empirical study of the citation distribution. Eur Phys J B. 1998;4(2):131-34.

5. Radicchi F, Fortunato S, Castellano C. Universality of citation distributions: Toward an objective measure of scientific impact. Proc Nat Acad Sci. 2008;105(45):17268-72.

6. Newman MEJ. Power laws, Pareto distributions and Zipf's law. Contemporary Physics. 2005;46(5):323-51.

7. Rousseau R. Double exponential models for first-citation processes. Scientometrics. 1994;30(1):213-27.

8. Egghe L. A Heuristic Study of the First-Citation Distribution. Scientometrics. 2000;48(3):345-59.

9. Burrell QL. Stochastic modelling of the first-citation distribution. Scientometrics. 2001;52(1):3-12.

10. Burrell QL. The $n$ th-citation distribution and obsolescence. Scientometrics. 2002;53(3):309-23.

11. Petersen AM, Stanley HE, Succi S. Statistical regularities in the rank-citation profile of scientists. Scientific Reports. 2011;1:181.

12. Katz JS. Scale-independent indicators and research evaluation. Science and Public Policy. 2000;27(1):23.
13. 13. Eom $\mathrm{YH}$, Fortunato $\mathrm{S}$. Characterizing and modeling citation dynamics. PloS one. 2011;6(9):e24926.

14. Garfield E. Science Citation Index-A new dimension in indexing. Science. 1964;144(3619):649-54.

15. Garfield E. Commentary: Fifty years of citation indexing. Int J Epidemiology. 2006;35(5):1127-8.

16. Bergstrom CT, West JD, Wiseman MA. The Eigenfactor ${ }^{\mathrm{TM}}$ metrics. J Neuroscience. 2008;28:11433.

17. Leydesdorff L, Bornmann L. Integrated impact indicators compared with impact factors: An alternative research design with policy implications. Journal of the American Society for Information Science and Technology. 2011;62(11):2133-46.

18. Fersht A. The most influential journals: Impact Factor and Eigen factor. Proc Nat Acad Sci. 2009;106(17):6883-4.

19. Thomson reuter, journal citation reports. http://admin-apps. webofknowledge.com/JCR/JCR?RQ=HOME.

20. Thompson reuters, essential science indicators. http://ip-science. thomsonreuters.com $/ \mathrm{m} / \mathrm{pdfs} / \mathrm{mgr} / \mathrm{qrc}$ esi mar09.pdf.

21. Redner S. Citation statistics from 110 years of physical review. Phys Today. 2005;58;49.

22. Chatterjee A, Mitrović M, Fortunato S. Universality in voting behavior: an empirical analysis. Scientific reports. 2013;3:1049.

How to cite this article: Khaleque A, Chatterjee A, Sen P. Annual journal citation indices: a comparative study. J Scientometric Res. 2016;5(1):25-33. 